Check for updates

Received 16th March 2018

Accepted 8th May 2018

DOI: $10.1039 / c 8 r a 02325 e$

rsc.li/rsc-advances
Cite this: RSC Adv., 2018, 8, 17443

\section{Wet-spun graphene filaments: effect of temperature of coagulation bath and type of reducing agents on mechanical \& electrical properties}

\begin{abstract}
Melik Oksuz and H. Yildirim Erbil (D) *
Although many factors are considered to improve the properties of graphene filaments, there is no report in the existing literature on the effect of the temperature of the coagulation bath to the mechanical properties of graphene oxide filaments obtained in the wet-spinning process and also to the mechanical and electrical properties of the resulting graphene filaments after reduction. In this study, the effect of the temperature of the isopropanol coagulation bath during wet-spinning of graphene filaments on their final properties after formation was investigated and it was found that the decrease of the coagulation bath temperature resulted in more compact filaments having better mechanical properties for both graphene oxide and corresponding reduced graphene filaments. The best tensile strength and Young's modulus values were obtained in isopropanol coagulation bath which was kept at $15{ }^{\circ} \mathrm{C}$. On the other hand, the types of the chemical reduction agents which can provide better electrical conductivity to graphene filaments after reduction were also investigated and it was determined that the use of hydriodic acid/acetic acid mixture resulted in graphene filaments having the best electrical conductivity $\left(1.28 \times 10^{4} \mathrm{~S} \mathrm{~m}^{-1}\right)$ and also tensile strength $(234 \pm 26 \mathrm{MPa}$ ) values. The addition of acetic acid into hydriodic acid increased the tensile strength $26 \%$ when compared with the plain $\mathrm{HI}$ treatment. Both electrical conductivity and tensile strength results were higher than most of the previously reported values of the wet-spun neat graphene filaments in the literature.
\end{abstract}

\section{Introduction}

2D graphene materials have many extraordinary properties such as very high electrical and thermal conductivity and high tensile modulus values. ${ }^{1-3}$ Graphene can be synthesized in many forms such as 2D large area thin films on substrates, films on paper, 3D aerogels, fibers etc. which can be used in many industrial applications. ${ }^{4-10}$ Graphene filaments (GFs) were first synthesized by Gao and $\mathrm{Xu}$ in 2011. ${ }^{7}$ These filaments are electrically conductive and proposed to be utilized in the production of light-weight motors,$^{11}$ supercapacitors ${ }^{12-17}$ and wearable smart textiles. ${ }^{18-20}$ GFs are flexible, low density materials having good thermal, electrical conductivity properties and attachment of functional groups onto them is easy. ${ }^{11-22}$

Two methods are generally used to obtain GFs: (i) wetspinning of an aqueous graphene oxide (GO) dispersion into a coagulation bath to obtain GO filaments which are then reduced to GFs by several methods; (ii) application of a hydrothermal reaction where an aqueous GO dispersion is injected into a glass tube and then subjected to thermal annealing

Department of Chemical Engineering, Gebze Technical University, Gebze 41400, Kocaeli, Turkey.E-mail: yerbil@gtu.edu.tr $\left(\sim 230{ }^{\circ} \mathrm{C}\right)$ to obtain the GF directly. ${ }^{23}$ This method can be also performed at low temperature $\left(\sim 80^{\circ} \mathrm{C}\right)$ in a plastic tube where a chemical reduction agent is added into GO dispersion before injection. ${ }^{24}$ It was reported that GFs obtained by the hydrothermal method had poor mechanical properties and lower electrical conductivity than that of the GFs obtained by the wetspinning method. Hydrothermal method was also found to be less suitable for large scale filament production..$^{11,23,24}$ Some other methods, such as chemical vapor deposition, ${ }^{25}$ electrophoretic self-assembly ${ }^{26}$ have also been reported in the literature to obtain GFs but they were reported to be more expensive when compared with the wet-spinning method.

Some additives were used to improve the properties of the resultant GFs. For example, Xu et al. obtained stretchable conductor GFs by adding silver nanowires to GO dispersion. ${ }^{27}$ Fang et al. produced hybrid fiber having fire-resistant properties by adding montmorillonite to system. ${ }^{28} \mathrm{Wu}$ et al. introduced hydrophilicity and antifouling property to polymeric membranes by using $\mathrm{GO} /$ water as coagulation bath. ${ }^{29}$

$\mathrm{Cao}$ and $\mathrm{Xu}$ reported that wet spun GFs production can be made by only using the GO dispersions having the liquid crystal property which can be obtained only at high GO concentrations in water. ${ }^{30}$ Thus, the increase of GO concentration in water 
dispersion by centrifuging is a necessary step before the wetspinning. There are mainly two stages in the wet-spinning process: (i) concentrated GO dispersion is injected into a coagulation bath to form the graphene oxide filaments (GOFs), then these filaments are removed from the bath and dried; (ii) dried GOFs are reduced via thermal annealing or with chemical reduction methods into GFs which are electrically conductive and mechanically strong enough to be used in weaving.

At present, wet-spinning method to obtain GFs is the most popular method as displayed in the increasing number of publications using this process by several groups worldwide. It was determined that the properties of the final GFs are directly affected by the properties of used GOFs. ${ }^{31-34}$ On the other hand, the type of the coagulation bath which is used in wet-spinning is another important parameter for the GOFs product. Although many different chemicals such as amine solutions, ${ }^{35}$ sulfuric acid and chitosan solution ${ }^{36}$ were reported to be used as the coagulation bath during wet-spinning, only three types of coagulation baths were commonly preferred in most of the publications. These are aqueous ionic $\left(\mathrm{Na}^{+}, \mathrm{K}^{+}, \mathrm{Ca}^{++}, \mathrm{Mg}^{++}\right.$, $\mathrm{Cu}^{++}$) solutions ${ }^{7,33,36}$ aqueous cationic surfactant (CTAB) solutions ${ }^{\mathbf{8 , 3 4}}$ and some organic solvents like ethyl acetate, methyl acetate, isopropanol, ether and acetone..$^{13,27,32,36}$

When ionic or surfactant containing coagulation baths are used then the obtained GOFs have to be kept in the bath for long durations $(\sim 30 \mathrm{~min})$ to complete the inter-diffusion process of ions and surfactant molecules between the GO layers in the filaments during self-assembly process. ${ }^{3,34,37}$ The interactions between GO platelets are weak for short filament immersion times and the resultant GOFs have poor mechanical properties. Another problem is the presence of the cationic surfactant molecules in the final GFs, which affects their electrical conductivity adversely. It was reported that the use of organic solvents as coagulation baths resulted in GFs having good mechanical and electrical properties. ${ }^{11,13,32}$ As a consequence of these experimental findings, only solvent based coagulation baths were used to produce GFs in a pilot plant study. ${ }^{11}$

Many parameters affecting the mechanical properties of GOFs and GFs were investigated previously in the literature and it was determined that the increase of the GO concentration in the aqueous dispersion resulted in an increase of the mechanical resistance of the obtained GOFs due to better interaction between the GO layers in a confined volume. The use of graphite powders having large particle sizes as the starting raw material for the GO production resulted in mechanically more resistant and electrically conducting GFs when compared with the GFs obtained by using graphite powders having small particle sizes. The presence of large interaction areas between the large GO platelets forms strong filaments. ${ }^{31}$ On the contrary, some authors suggested that better mechanical properties of GFs could be obtained by mixing both small (30\%) and large (70\%) GO particles in the doping GO dispersion, since small GO plates would fill the voids between the large ones during the GO filament formation process. ${ }^{34}$ On the other hand, it was also reported that the mechanical and electrical properties of the filaments could be improved by the application of an optimum tension to GOFs during their production in the coagulation bath. ${ }^{11,32,33}$

Many factors have been considered to improve the properties of neat GFs when the wet-spinning system was used up to now. However, there was no report in the existing literature on the effect of the temperature of the coagulation bath to the mechanical and electrical properties of GOFs and GFs in the wet-spinning process to the best of our knowledge. It is hypothesized that the effect of temperature during the coagulation step should be an important parameter in the wetspinning process to affect the mechanical properties of GOFs since it will change the inter-diffusion rate between the water molecules present in the GO dispersion and solvent molecules in the bath and thus will affect the GO platelet self-assembly process. In this work, we investigated the effect of the temperature of an organic solvent (isopropanol) coagulation bath to the mechanical properties of GOFs and, also to the electrical and mechanical properties of GFs obtained after reducing these GOFs chemically. It was determined that the decrease of the coagulation temperature resulted in better mechanical properties for both GOFs and GFs. In addition, we investigated the effect of the types of the chemical reduction agents to improve the electrical properties of GFs and found that the use of hydriodic acid/acetic acid mixture resulted in the GFs having the best electrical and mechanical properties.

\section{Experimental}

\section{Materials}

Graphite flakes (ca. $150 \mu \mathrm{m}$, product no: 332461), hydriodic acid (HI, 57 wt\%), acetic acid (AAc) and hydrazine monohydrate (Hdz) were purchased from Sigma Aldrich. Potassium permanganate $\left(\mathrm{KMnO}_{4}\right)$, hydrogen peroxide $\left(\mathrm{H}_{2} \mathrm{O}_{2}, 35 \mathrm{wt} \%\right)$, sulfuric acid $\left(\mathrm{H}_{2} \mathrm{SO}_{4}, 98 \mathrm{wt} \%\right)$, phosphoric acid $\left(\mathrm{H}_{3} \mathrm{PO}_{4}, 85 \mathrm{wt} \%\right)$ and ascorbic acid (AsAc) were purchased from Merck. Technical grade isopropanol was purchased from Balmumcu Chemicals, Turkey. All materials were used as received without further purification.

\section{Graphene oxide synthesis}

A modified Tour method was used for the graphene oxide (GO) synthesis in this study. ${ }^{38,39}$ Initially, graphite flakes $(5 \mathrm{~g})$ was added into a 5 : 1 mixture of $\mathrm{H}_{2} \mathrm{SO}_{4}(200 \mathrm{~mL}) / \mathrm{H}_{3} \mathrm{PO}_{4}(40 \mathrm{~mL})$ and

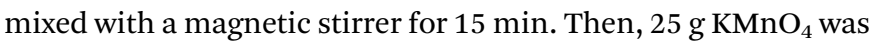
slowly dissolved in the acid-graphite mixture, which were cooled to $10{ }^{\circ} \mathrm{C}$ with the aid of a cooling jacket to avoid the explosion risk for $75 \mathrm{~min}$. After the addition of $\mathrm{KMnO}_{4}$, the green mixture was heated up to $40{ }^{\circ} \mathrm{C}$ to carry out the oxidation of graphite under stirring in a water bath. After two hours, the mixture turns into a viscous slurry, which was stirred for a further 2.5 hours by using a Teflon rod (in every $7 \mathrm{~min}$ ). A viscous brown mixture was obtained after 4.5 hours of the oxidation reaction and cooled down to room temperature. Later, a mixture of $600 \mathrm{~mL}$ of cold water with $40 \mathrm{~mL}$ of $35 \mathrm{wt} \%$ $\mathrm{H}_{2} \mathrm{O}_{2}$ was slowly added into the viscous mixture and the color of mixture turned from brown to yellow during this stage. The final 
mixture was left overnight at room temperature and then, GO was separated from the mixture with the aid of a centrifuge at $13500 \mathrm{rpm}$. GO cake was washed with $1 \mathrm{~L} \mathrm{H}_{2} \mathrm{SO}_{4}$ solution (5 wt\%) and centrifuged repeatedly after washing with water until the $\mathrm{pH}$ value of the supernatant was $\sim 5$. Finally, pure GO cake was dispersed in $2 \mathrm{~L}$ of distilled water and aqueous GO dispersion was centrifuged at $3000 \mathrm{rpm}$ for $15 \mathrm{~min}$ to separate un-exfoliated graphite oxide and un-oxidized graphite particles. Aqueous GO dispersion (supernatant) was collected in a flask after centrifugation and stored till use for wet-spinning. $1.40 \pm$ $0.05 \mathrm{~g}$ of GO per $\mathrm{g}$ graphite was obtained with this synthesis procedure.

\section{Wet-spinning of GO filaments}

GO dope (13 $\mathrm{mg} \mathrm{mL}^{-1}$ ) were prepared from the aqueous GO dispersion by centrifugation at $13500 \mathrm{rpm}$. A wet-spinning system with a rotating bath was used for the production of GO filaments (Fig. 1). GO dope was injected into the coagulation bath at a rate of $0.25 \mathrm{~mL} \mathrm{~min}^{-1}$ through a motorized nozzle having an inner diameter of $440 \mu \mathrm{m}$. Coagulation bath was rotated at a speed of $12 \mathrm{rpm}$ during the GO injection. The nozzle was about $3 \mathrm{~cm}$ away from the center of rotating bath. Isopropanol and acetone solvents were used as coagulation baths at room temperature. In further experiments, isopropanol temperature was kept constant at 5, 15, 25 and $35^{\circ} \mathrm{C}$ in order to investigate effect of the temperature of the coagulation bath on the mechanical properties of wet-spun GOFs. These experiments were performed in a climatic chamber which can cool down to $-30{ }^{\circ} \mathrm{C}$ (Memmert CTC 256), to avoid temperature changes (Fig. 1). GO filaments were kept immersed in the coagulation bath for only $2 \mathrm{~min}$, then removed from the bath and placed horizontally on a polypropylene frame where they

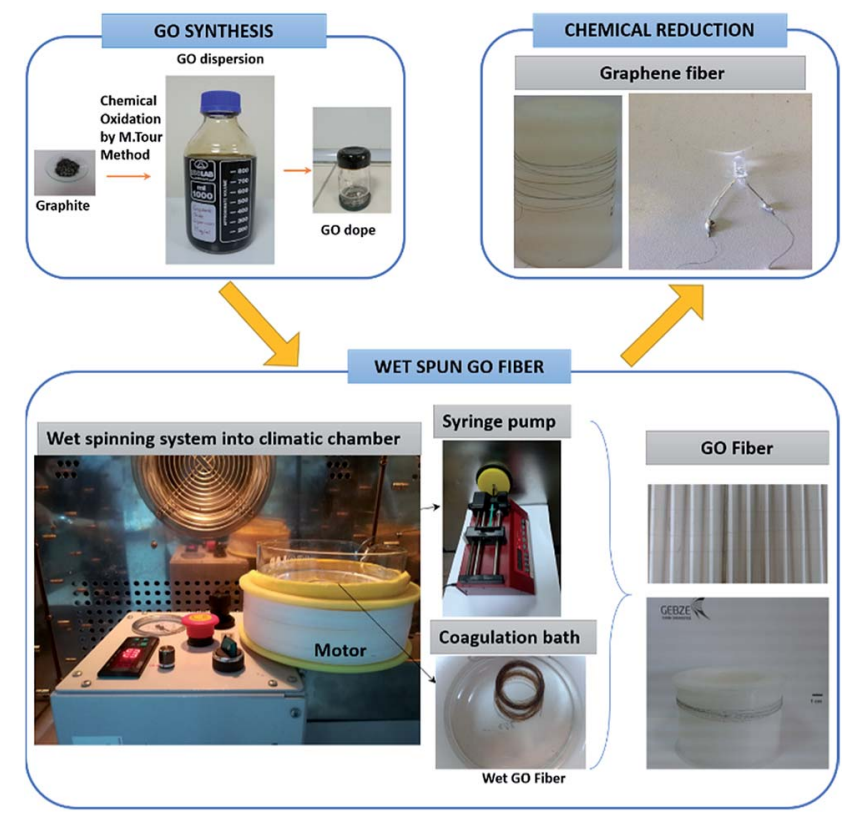

Fig. 1 Wet-spinning system to obtain GOFs and GF filament from graphite. are adhered with a pressure sensitive tape after slightly straightening and dried at room temperature.

\section{Reduction of GO filament into graphene filament}

Dried GOFs were reduced into GFs by using different chemical reduction agents including (i) ascorbic acid (AsAc), (ii) hydrazine monohydrate (Hdz), (iii) hydriodic acid (HI) and (iv) hydriodic acid/acetic acid (HI/AAc) mixture at temperatures ranging between $40-90{ }^{\circ} \mathrm{C}$. Details of the reduction methods are given below: (i) AsAc reduction: ${ }^{27,40} \mathrm{GO}$ filaments were kept in an aqueous solution of AsAc $(0.1 \mathrm{M})$ at $90{ }^{\circ} \mathrm{C}$ for $16 \mathrm{~h}$; (ii) $\mathrm{Hdz}$ reduction: ${ }^{41}$ GO filaments were placed into a glass bottle and $200 \mu \mathrm{L}$ of $\mathrm{Hdz}$ (98 wt\%) was dropped into the bottle rapidly and the bottle temperature was increased up to $80{ }^{\circ} \mathrm{C}$ and kept in these conditions for $24 \mathrm{~h}$; (iii) HI reduction: ${ }^{42} \mathrm{GO}$ filaments were kept in an aqueous HI solution ( $57 \mathrm{wt} \%)$ at $80{ }^{\circ} \mathrm{C}$ for $16 \mathrm{~h}$; (iv) HI/AAc reduction: ${ }^{43}$ GO filaments were horizontally placed in a glass desiccator $(1.7 \mathrm{~L})$ containing a mixture of $2 \mathrm{~mL}$ of $\mathrm{HI}$ (57 wt\%) and $5 \mathrm{~mL}$ of pure AAc and kept at $40{ }^{\circ} \mathrm{C}$ in the desiccator for $24 \mathrm{~h}$. After reduction, GFs were washed by water and methanol and dried at room temperature. When HI/AAc mixture was used as the reduction agent, the filaments were initially washed with sodium bicarbonate solution (5 wt\%) before water and methanol washing.

\section{Characterization}

X-ray diffraction (XRD) patterns of GO were obtained with a Rigaku $\left(D_{\max } 2200\right)$ diffractometer using $\mathrm{CuK} \alpha$ radiation $(\lambda=$ $0.154 \mathrm{~nm}$ ) at $40 \mathrm{kV}$. Differential scanning calorimeter (DSC) analysis of GO was performed with a Perkin-Elmer Jade instrument at a heating rate of $5{ }^{\circ} \mathrm{C} \mathrm{min}^{-1}$ under nitrogen gas flow. Attenuated total reflectance-Fourier transform infrared (ATRFTIR) spectra of GO were recorded with a Perkin-Elmer Spectrum 100. Polarized optical microscopy observations of concentrated GO dope were performed with a Leitz Orthoplan instrument. Morphologies of GOFs and GFs were examined by scanning electron microscopy (SEM, Philips XL 30 SFEG). Mechanical tests (tensile strength, Young's modulus, elongation at break) of both GOFs and GFs were performed in a universal tensile tester (Devotrans BP D-NN, Turkey) equipped with a $10 \mathrm{~N}$ load cell, at a strain rate of $0.5 \mathrm{~mm} \mathrm{~min}^{-1}$. Paper frames were initially prepared similar to ASTM D3379-75 method and the filaments were attached to the frames by using double sided tape to prevent the slippage between the jaws during the tensile tests (Fig. 3a). Sides of the paper frames were cut with a scissor before the test to eliminate any pre-tension effects on the measured force. The gauge length was $20 \mathrm{~mm}$. Cross sectional area of filaments after the fracture were determined by using an optical microscope (Nikon Eclipse LV 100D) using the Clemex software. Measured cross sectional areas of filaments were used in the calculation of the tensile strength per unit area and Young's modulus. At least five different samples were used for each tensile test and the results were reported as the arithmetic average of five measurements. Electrical conductivities of graphene filaments were measured with a four-probe method by using a source-meter (Keithley 2400). GFs were fixed on an 
electrically insulating glass slide using solder having a conductivity of $3 \times 10^{5} \mathrm{~S} \mathrm{~m}^{-1}$ and probes were contacted to the center of the solders on the filaments. Measurements were repeated on at least three times for each filament. Finally, the electrical conductivity values of GFs calculated from measured electrical resistance $(R)$, cross-section area $(A)$, and the length $(L)$ of filament sample (conductivity $=L / R A$ ).

\section{Results and discussion}

\section{Properties of the synthesized GO and presence of the liquid} crystallinity in the concentrated GO dope

A sharp peak of graphene oxide is seen in the XRD pattern at $2 \theta=9.56^{\circ}$ (Fig. 2a). Layer-to layer distances between the graphene plates in the graphite raw material was initially $3.37 \AA$ and increased up to $9.24 \AA$ after the oxidation step because of the introduction of epoxide, hydroxyl, carbonyl and carboxyl groups. ${ }^{38,43,46} \mathrm{XRD}$ patterns indicate the successful oxidation of the graphene platelets. GO showed an exothermic peak at ca. $220^{\circ} \mathrm{C}$ in the DSC curve (Fig. 2b) due to desorption of oxygen groups indicating their previous existence in the GO sample. The presence of carboxyl and hydroxyl groups are also seen in the FTIR-ATR spectra (O-H peaks in the $3300-3600 \mathrm{~cm}^{-1}$ region). In addition, the characteristic peaks of the $\mathrm{C}=\mathrm{O}$ groups in the $\sim 1720 \mathrm{~cm}^{-1}$ region, $\mathrm{C}-\mathrm{O}-\mathrm{C}$ groups in the $c a .1370 \mathrm{~cm}^{-1}$ region, $\mathrm{C}-\mathrm{O}$ groups in the $c a$. $1050 \mathrm{~cm}^{-1}$ region and $\mathrm{C}=\mathrm{C}$ groups in the $1620 \mathrm{~cm}^{-1}$ region show the presence of hydroxyl, epoxide, carbonyl and carboxyl groups and also the un-oxidized $\mathrm{C}=\mathrm{C}$ bonds. The presence or absence of the liquid crystallinity was checked by using a Polarized Optical
Microscope (POM) and it was determined that concentrated aqueous GO dope (13 $\mathrm{mg} \mathrm{mL}^{-1}$ ) has liquid crystalline properties. An indicative POM image of the GO dope is shown in Fig. 2d where the orientation of the GO plates and the double diffracted structures are easily seen to demonstrate the presence of liquid crystallinity.

Effect of the coagulation bath type on mechanical properties of wet-spun graphene oxide filaments

As mentioned in the introduction section, the type of the coagulation bath is a very important parameter to affect the mechanical properties of the resultant GOFs in the wetspinning process. The inter-diffusion rate between water molecules present in the injected GO dispersion and solvent molecules in the coagulation bath affects GO platelet selfassembly mechanism in the filament and thus the corresponding mechanical properties. It is well-known that the tensile properties of both GOFs and the accompanying GFs after the reduction step depends on this self-assembly process. ${ }^{32}$ In our study, acetone and isopropanol (IPA) solvents were used as coagulation baths at room temperature and the mechanical properties of GOFs were measured after drying of the filaments.

Acetone is one of the most commonly used solvent to remove water from the GO filament during the production of wet spun GFs at much higher rates compared to other solvents given in the literature..$^{13,18,36}$ In this study, IPA was used to remove water from the filament with a much lower rate compared to acetone and it was found that GOFs which were obtained in an IPA bath
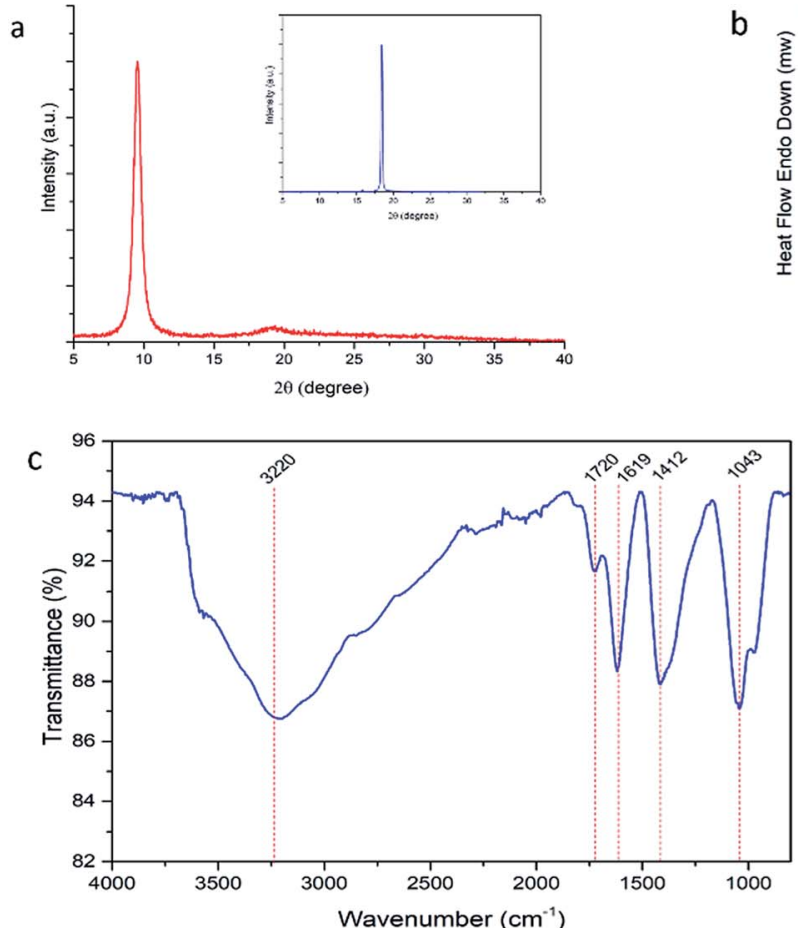
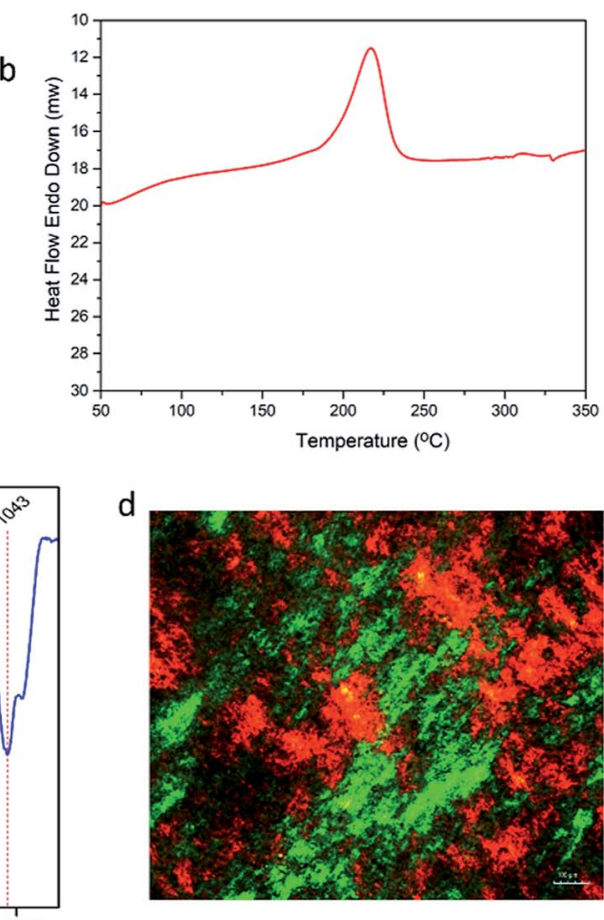

Fig. 2 (a) XRD patterns of graphene oxide, inset: XRD pattern of graphite; (b) DSC curve of GO (c) FTIR-ATR spectra of GO; (d) POM images of GO dope. 
resulted in higher tensile strength and smaller cross-sectional areas (giving more dense filaments) than that of the filaments obtained from the acetone bath as seen in Table 1. It is possible that low tensile strength GOFs were produced when acetone was used as the bath because of the more rapid inter-diffusion rate between water and acetone molecules than that of the interdiffusion rate between water and isopropanol molecules. The increase in the mass transfer rate resulted in filaments having longer spacing between GO platelets showing poor mechanical properties. $^{32}$ Indeed, the larger cross-sectional areas of GOFs obtained by the acetone coagulation bath showed a more loose filament structure. Consequently, IPA coagulation baths which gives mechanically stronger GOFs were used for the subsequent bath temperature effect experiments.

\section{Effect of the temperature of the IPA coagulation bath on the mechanical properties of wet-spun graphene oxide filaments}

IPA coagulation bath temperature was kept constant at different temperatures of $5,15,25$ and $35{ }^{\circ} \mathrm{C}$ during the injection of the GO dope (13 $\mathrm{mg} \mathrm{mL}^{-1}$ ) in order to investigate the effect of the bath temperature on the mechanical properties of wet-spun GOFs. The codes of the resultant GO filaments are given as: GOF@5 -IPA; GOF@15-IPA; GOF@25-IPA; GOF@35-IPA. It was previously reported in the literature that the actual filament cross-sectional areas after the rupture were smaller than the areas measured from the initial cylindrical cross-sections before the tensile tests. In this work, only actual cross-sectional areas were used for the tensile test calculations. ${ }^{\mathbf{1 1 , 3 6}}$ Irregular crosssectional filament areas were calculated from SEM and optical microscope images (Fig. 3b) of the GO filaments after rupture in the tensile tests. Filament tensile strength and Young's modulus values were determined by using these actual crosssectional areas and it was found that these areas were approximately $60 \%$ of the initial cylindrical areas in most of the cases (Fig. 3b).

Cross-section areas, tensile strengths and elongation at break values of GOFs are given in Table 2. It was determined that better filament tensile strengths and Young's modulus values were obtained when cold isopropanol coagulation baths were used. It is possible that the decrease in the bath temperature resulted in a slower inter-diffusion rate between water and isopropanol molecules and the decrease in the mass transfer rate produces GOFs having less spacing between the graphene oxide platelets in the filament. The contraction in the filament structure resulted in improved mechanical properties. Similar findings were also reported in the published works which were investigating the effect of temperature of coagulation baths on the mechanical properties and morphology of polyacrylonitrile

Table 1 Mechanical properties of GOFs produced by using different solvents

\begin{tabular}{llll}
\hline $\begin{array}{l}\text { Coagulation } \\
\text { bath solvent }\end{array}$ & $\begin{array}{l}\text { Cross section } \\
\text { area }\left(\mu \mathrm{m}^{2}\right)\end{array}$ & $\begin{array}{l}\text { Tensile strength } \\
(\mathrm{MPa})\end{array}$ & $\begin{array}{l}\text { Elongation } \\
\text { at break }(\%)\end{array}$ \\
\hline $\begin{array}{l}\text { Acetone } \\
\text { Isopropanol }\end{array}$ & $2281 \pm 91$ & $124 \pm 13$ & $5.0 \pm 1.2$ \\
2194 \pm 294 & $156 \pm 15$ & $5.2 \pm 0.6$
\end{tabular}
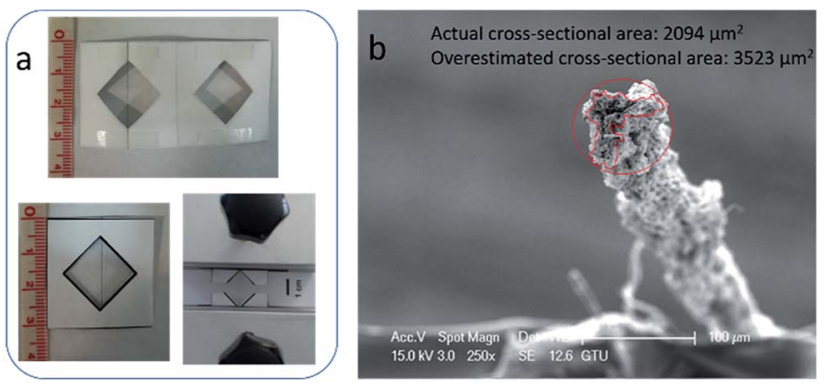

Fig. 3 (a) The paper frames used during the tensile tests; (b) SEM image of GOF $225^{\circ}$-IPA sample after the rupture during the tensile test to calculate their cross-section.

fibers. ${ }^{\mathbf{4 4 , 4 5}}$ As seen in Table 2, the cross-sectional areas deceased with the decrease of the bath temperature indicating the compact structure of GOFs after wet-spinning.

SEM images of the surfaces of GOFs under different magnifications are given in Fig. 4. The GOFs sample obtained from the coagulation bath having the highest temperature (GOF@35 ${ }^{\circ}$-IPA) had a very loose filament structure with the widest diameter and largest cross-sectional area, whereas the GOFs sample obtained with the lowest bath temperature (GOF@5 $5^{\circ}$-IPA) had a very contracted filament structure having the smallest cross-sectional area as seen in Fig. 4 and Table 2. It was found that the structural properties of GOFs affected to their tensile strength results. The most compact filament structure resulted in the highest tensile strength and Young's modulus value indicating that the use of coldest IPA coagulation bath should be favored to obtain the best mechanical properties. However, GOF@5 ${ }^{\circ}$-IPA sample was an exception since its mechanical properties, especially tensile strength (161 $\pm 11 \mathrm{MPa}$ ), was slightly lower than that of the GOF@15 -IPA sample (tensile strength $=181 \pm 23 \mathrm{MPa}$ ) although the bath temperature was $10^{\circ}$ higher. A possible explanation for this point is that the inter-diffusion rate during water-isopropanol exchange was very slow at $5{ }^{\circ} \mathrm{C}$ and our constant immersion duration of $2 \mathrm{~min}$ in the coagulation bath at this temperature was not enough to finish the GO platelet assembly process in the filament. In order to check the validity of this idea that the constant 2 min period may not be enough for the $5{ }^{\circ} \mathrm{C}$ experiments, we extended the immersion time up to $15 \mathrm{~min}$ in IPA and obtained GOFs having much better mechanical properties (tensile strength: $189 \pm 26 \mathrm{MPa}$, elongation at break: \% $5.1 \pm 0.9$ and Young's modulus $=18 \pm 3 \mathrm{GPa}$ ). This result indicates that a duration of two min is not enough for the $5{ }^{\circ} \mathrm{C}$ experiments. However, the increase of filament immersion time in the bath is generally a disadvantage for a commercial continuous wetspinning process. For this reason, we selected GOF@15 ${ }^{\circ}$-IPA sample, which was kept only for $2 \mathrm{~min}$ at $15{ }^{\circ} \mathrm{C}$ in the bath to have the optimum mechanical properties and can be used for any scale-up experiments.

\section{Effect of type of reducing agents on the mechanical properties and electrical conductivities of wet-spun graphene filaments}

GOFs which are obtained by the wet-spinning method are not electrically conductive due to the presence of oxygen containing 
Table 2 Mechanical Properties of GOFs obtained in IPA coagulation bath at different temperatures

\begin{tabular}{|c|c|c|c|c|}
\hline Sample & $\begin{array}{l}\text { Cross section } \\
\text { area }\left(\mu \mathrm{m}^{2}\right)\end{array}$ & $\begin{array}{l}\text { Tensile strength } \\
\text { (MPa) }\end{array}$ & $\begin{array}{l}\text { Elongation at } \\
\text { break }(\%)\end{array}$ & $\begin{array}{l}\text { Young's } \\
\text { modulus (GPa) }\end{array}$ \\
\hline GOF@5-IPA & $1962 \pm 142$ & $161 \pm 11$ & $5.2 \pm 0.5$ & $15.4 \pm 4.4$ \\
\hline GOF@25-IPA & $2194 \pm 294$ & $156 \pm 15$ & $5.2 \pm 0.6$ & $14.1 \pm 3.7$ \\
\hline GOF@35-IPA & $2315 \pm 126$ & $147 \pm 9$ & $5.2 \pm 0.4$ & $12.0 \pm 3.3$ \\
\hline
\end{tabular}

epoxide, hydroxide, carbonyl and carboxyl groups in their structure and must be chemically reduced to obtain GFs by removing all of these oxygen containing chemical groups. Hydriodic acid, ascorbic acid and hydrazine monohydrate are some examples which are used in the existing wet-spun filament literature for this purpose. ${ }^{8,27,36}$ In our study, AsAc, HI, Hdz and $\mathrm{HI} / \mathrm{AAc}$ mixture were tested as chemical reducing agents on the same GOFs samples to obtain best mechanical and electrical conductivity results for the resultant GFs. HI/AAc mixture was previously used to reduce a GOF sample containing polymers ${ }^{47}$ and we used it for the first time to reduce the neat GOFs. Tensile strength, Young's modulus and electrical conductivity results of the reduced GFs are given in Table 3.
It was found that graphene filaments having the best tensile strength and Young's modulus values were obtained from the dried GOF@25 -IPA filaments when HI/AAc mixture was used as the chemical reducing agent. A tensile strength value of $200 \pm$ 37 MPa was found which was higher than most of the previously reported GFs that was reduced by using only chemical

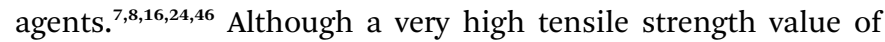
501 MPa was reported for GFs in the existing literature which was reduced by using only chemical agents, ${ }^{33}$ this method has some drawbacks since the filament immersion time in the bath (ca. $30 \mathrm{~min}$ ) is much longer than ours ( $2 \mathrm{~min}$ ).

GFs having the most porous filament structure and the worst mechanical properties were obtained when hydrazine
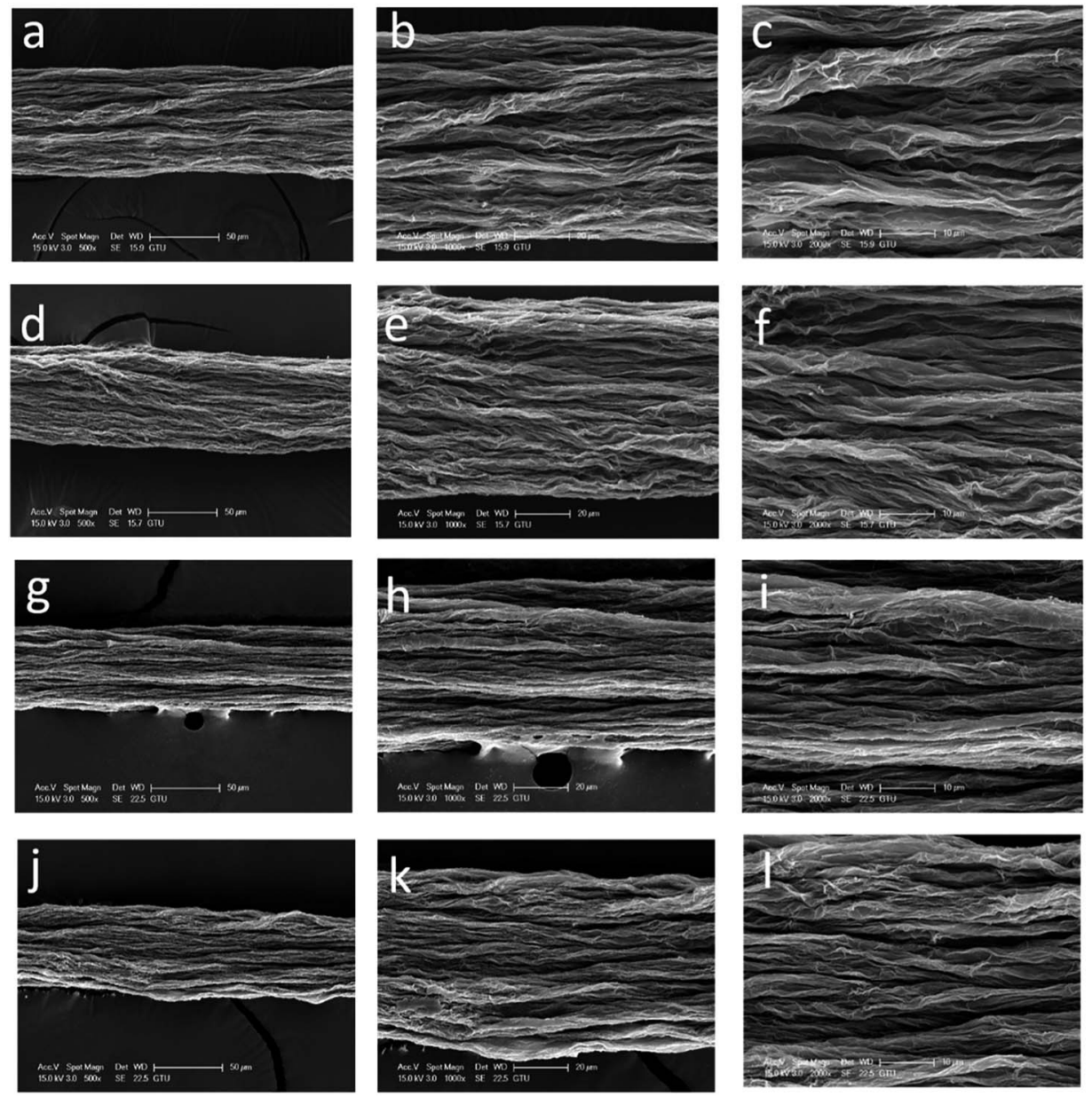

Fig. 4 SEM images of the surface of GOFs under different magnifications: (a-c) GOF@35 $5^{\circ}-I P A ;(d-f) G O F\left(25^{\circ}-I P A ;(g-i) G O F @ 15^{\circ}-I P A ;(j-l)\right.$ GOFa5 $5^{\circ}$ IPA 
Table 3 Mechanical properties and electrical conductivity of graphene filaments obtained by using different chemical reduction agents using GOF@25-IPA samples

\begin{tabular}{lllllrr}
\hline $\begin{array}{l}\text { Chemical } \\
\text { reduction agent }\end{array}$ & $\begin{array}{l}\text { Reduction } \\
\text { temperature }\left({ }^{\circ} \mathrm{C}\right)\end{array}$ & $\begin{array}{l}\text { Cross section } \\
\text { area }\left(\mu \mathrm{m}^{2}\right)\end{array}$ & $\begin{array}{l}\text { Tensile strength } \\
(\mathrm{MPa})\end{array}$ & $\begin{array}{l}\text { Elongation at } \\
\text { break }(\%)\end{array}$ & $\begin{array}{l}\text { Young's } \\
\text { modulus }(\mathrm{GPa})\end{array}$ & $\begin{array}{l}\text { Electrical conductivity } \\
\left.(\mathrm{S} \mathrm{m})^{-1}\right)\end{array}$ \\
\hline Hydrazine monohydrate & 90 & $3184 \pm 19$ & $33 \pm 6$ & $2.4 \pm 1.4$ & $4.2 \pm 1.5$ & $0.09 \pm 0.03 \times 10^{4}$ \\
Hydriodic acid & 80 & $1537 \pm 143$ & $178 \pm 34$ & $6.3 \pm 0.8$ & $17.0 \pm 4.1$ & $0.90 \pm 0.05 \times 10^{4}$ \\
Ascorbic acid & 90 & $2279 \pm 48$ & $141 \pm 12$ & $4.2 \pm 2.3$ & $20.6 \pm 1.5$ & $0.24 \pm 0.02 \times 10^{4}$ \\
HI/AAc & 40 & $1471 \pm 115$ & $200 \pm 37$ & $5.7 \pm 0.9$ & $21.0 \pm 2.7$ & $1.20 \pm 0.07 \times 10^{4}$ \\
\end{tabular}

monohydrate was used as the reducing agent probably due to the adsorption of gases formed during the reduction reaction. ${ }^{47}$ On the contrary, the most compressed filament structure, giving the best mechanical properties was obtained when HI/AcA mixture was used as seen in SEM images (Fig. $5 a$ and b). It is interesting that the inclusion of acetic acid into hydriodic acid increased the tensile strength $26 \%$ when compared with the plain HI solution. Similarly, Young's modulus values of GFs increased by $24 \%$ after HI/AAc reduction as compared with HI. Other reducing agents resulted in more loose filament structures in the following order: $\mathrm{HI}<\mathrm{AsAc}<\mathrm{Hdz}$. The use of ascorbic acid also resulted in a loose GFs structure. Electrical conductivity results of GFs are given in Table 3 . The best result was obtained when HI/AAc mixture was used as the chemical reducing agent for the dried GOF@25 ${ }^{\circ}$-IPA filament $\left(1.20 \times 10^{4}\right.$ $\mathrm{S} \mathrm{m}{ }^{-1}$ ) which is much higher than most of the electrical conductivity values of $\left(0.03-0.86 \times 10^{4} \mathrm{~S} \mathrm{~m}^{-1}\right)$ of wet-spun neat GFs reported in the literature.,.$^{\mathbf{8}, 16,18,36}$ Very high electrical conductivity values ranging between $2.14 \times 10^{4}$ and $4.1 \times 10^{4} \mathrm{~S}$ $\mathrm{m}^{-1}$ were also reported in some publications where only chemical agents were used for the reduction..$^{7,27,33,46}$ Our results were close to these values and the addition of acetic acid into HI increased the electrical conductivity $33 \%$ when compared with the results obtained with plain HI. The worst electrical conductivity value was obtained when $\mathrm{Hdz}$ was used as the reducing agent. On the other hand, electrical conductivity values of GFs can reach up to $2-8 \times 10^{5} \mathrm{~S} \mathrm{~m}^{-1}$ when very high temperature thermal annealing $\left(1300-3000{ }^{\circ} \mathrm{C}\right)$ reduction process was used..$^{11,34,37}$ However, such thermal reductions are very costly requiring special furnaces and uses expensive inert gas atmospheres and consequently, chemical reduction is a better choice to avoid these high costs.

\section{Effect of IPA coagulation bath temperature on the mechanical} properties and electrical conductivities of graphene filaments

After we determined that the best performing reducing agent was HI/AAc, then used this mixture to examine the effect of IPA coagulation bath temperature on the mechanical properties and electrical conductivities of wet-spun GOFs under the same reduction conditions. The codes of the reduced graphene filaments after the HI/AAc reduction are given as: GF@5 $5^{\circ}$-IPA; GF@15-IPA; GF@25-IPA; GF@35 ${ }^{\circ}$-IPA. SEM images of GFs obtained by reducing the dried GOFs obtained by using IPA coagulation baths at different temperatures $\left(5,15,25\right.$ and $\left.35{ }^{\circ} \mathrm{C}\right)$ under different magnifications are given in Fig. 6. In general, all the GFs have much shorter filament diameters than that of GOFs and a very compressed structure after the reduction due to the removal of the most of the oxygen containing chemical groups attached to graphene platelets. Cross-sectional areas of GFs after reduction decreased by $30-35 \%$ in comparison with the corresponding GOFs. Cross-sectional areas of GF@ $5^{\circ}$-IPA,

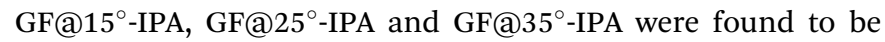
$1378 \pm 57,1385 \pm 92,1471 \pm 115$ and $1529 \pm 57 \mu^{2}$, respectively. It was determined that the effect of coagulation temperature to the GFs structure was similar, the cooler the bath
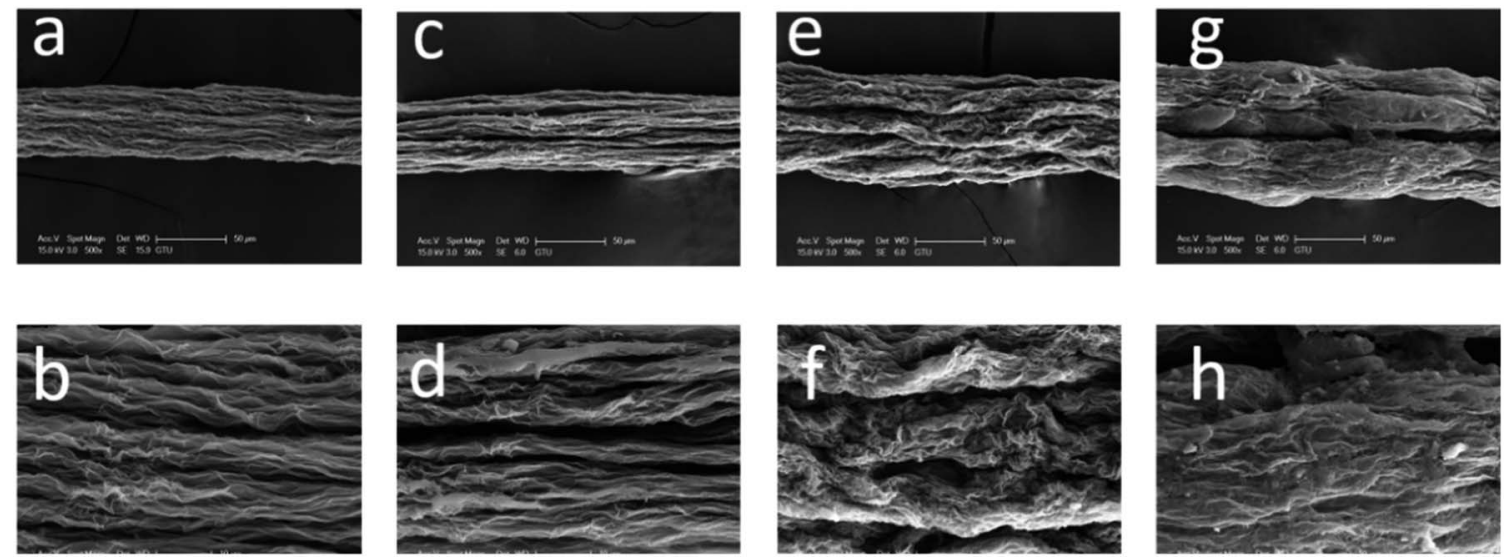

Fig. 5 SEM images of GFs obtained by reducing dried GOFa25-IPA by different chemical agents: $500 \times$ and $2000 \times$ magnification (a and b) reduced graphene filament by $\mathrm{HI} / \mathrm{AAc}$, (c and d) reduced graphene filament by $\mathrm{HI}$, (e and f) reduced graphene filament by AsAc, ( $\mathrm{g}$ and $\mathrm{h}$ ) reduced graphene filament by $\mathrm{Hdz}$. 

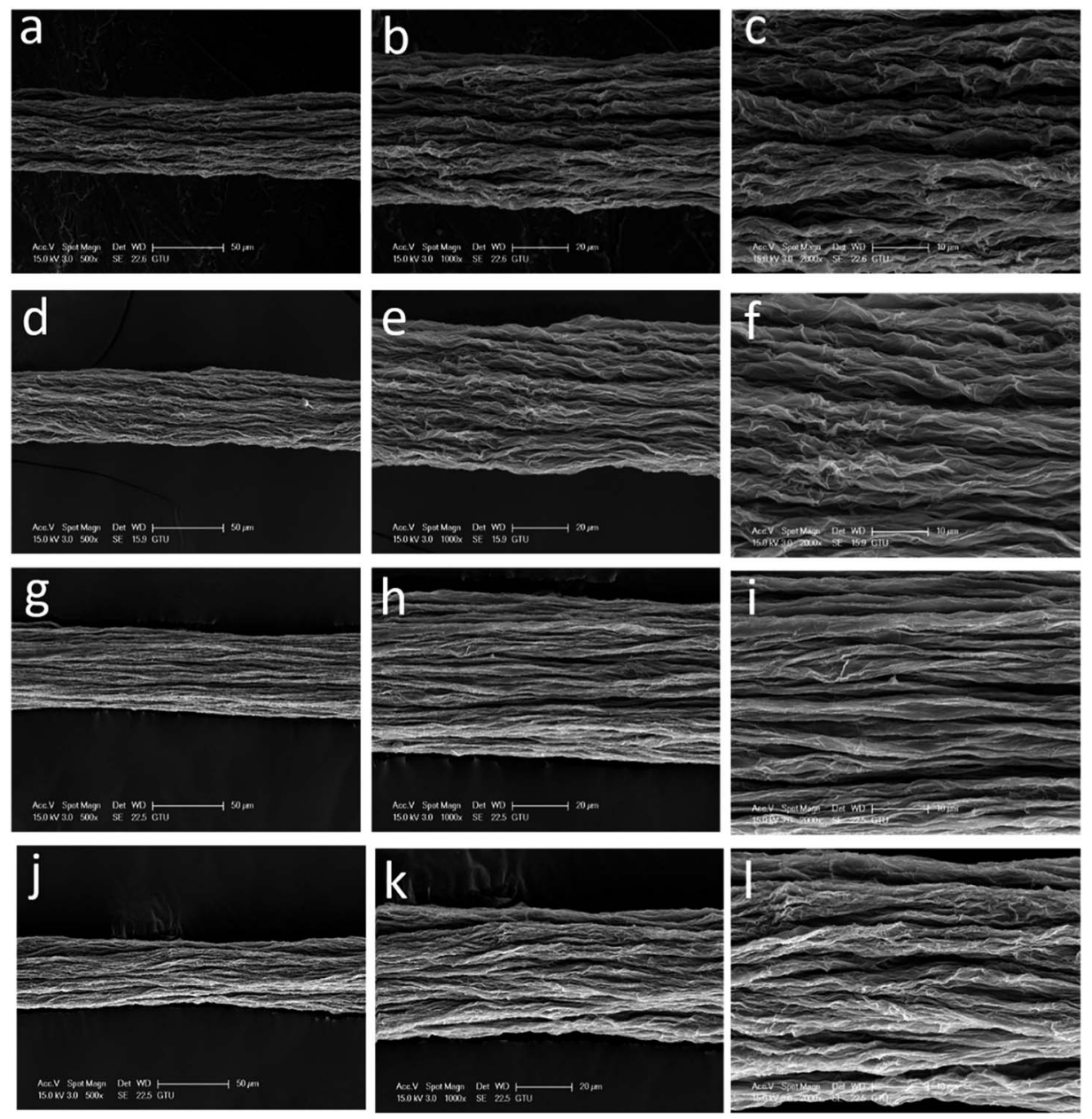

Fig. 6 SEM images of GFs obtained by reducing the dried GOFs obtained by using isopropanol coagulation baths at different temperatures under

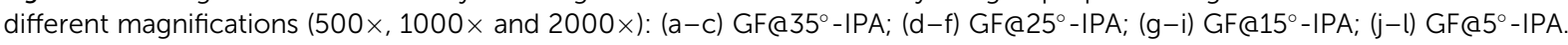

temperature, the smaller the GFs diameters and cross-sectional areas were obtained with a more compact structure as seen in Fig. 6.

Some typical stress-strain curves of GFs are shown in Fig. 7. The mechanical properties of GFs after reduction were much better than that of the mechanical properties of the initial GOFs. The best tensile strength and Young's modulus values were obtained with the reduced GOF@15 ${ }^{\circ}$-IPA sample, which was obtained in IPA bath at $15^{\circ} \mathrm{C}$ as seen in Table 4 . Both of the GF@25 -IPA and GF@3 $5^{\circ}$-IPA samples had good tensile strength values when compared with the literature values. Tensile strength values of 8-163 MPa and Young's modulus values of 1.9-10.7 GPa were reported previously., ${ }^{7,24,36,46}$ It was also realized that the tensile strength values of the resultant GFs were parallel to the tensile strength values of the corresponding GOFs, similar to some reports. ${ }^{31,33}$

The best electrical conductivity value of GFs was obtained to be $1.28 \times 10^{4} \mathrm{~S} \mathrm{~m}^{-1}$ with the reduced GOF@15-IPA sample as seen in Table 4. In general, it was determined that the effect of coagulation bath temperature on the electrical conductivities is not very important as seen in the close electrical conductivity results of the GFs samples obtained at different bath temperatures. In summary, we obtained GFs having good mechanical properties and electrical conductivities by using a coagulation bath of isopropanol at $15{ }^{\circ} \mathrm{C}$ and chemically reducing the graphene oxide filament at $40{ }^{\circ} \mathrm{C}$ using HI/AAc solution. The obtained GFs are suitable to light small bulbs (Fig. 1). It is well-known that very high tensile strength values of

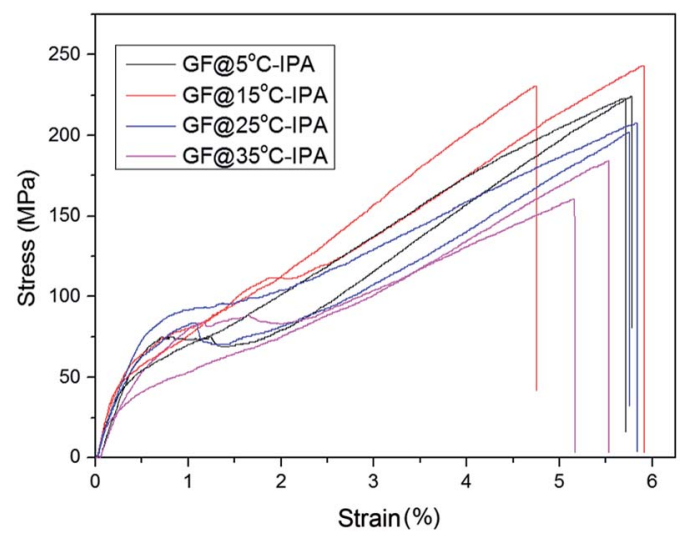

Fig. 7 Stress-strain curves of GOFs obtained in isopropanol at different temperatures. 
Table 4 Mechanical properties and electrical conductivity of graphene filaments obtained by HI/AAc reducing agent compared with the literature values

\begin{tabular}{|c|c|c|c|c|c|c|c|}
\hline GF@5-IPA & $\mathrm{HI} / \mathrm{AcA}$ & 40 & $218 \pm 24$ & $5.7 \pm 1.1$ & $19.3 \pm 4.0$ & $1.18 \pm 0.06 \times 10^{4}$ & This study \\
\hline GF@25-IPA & $\mathrm{HI} / \mathrm{AcA}$ & 40 & $200 \pm 37$ & $5.7 \pm 0.9$ & $21.0 \pm 2.7$ & $1.20 \pm 0.07 \times 10^{4}$ & This study \\
\hline GF@35-IPA & $\mathrm{HI} / \mathrm{AcA}$ & 40 & $176 \pm 33$ & $5.6 \pm 0.3$ & $16.2 \pm 3.0$ & $1.19 \pm 0.04 \times 10^{4}$ & This study \\
\hline GF & $\mathrm{HI}(40 \%)$ & 80 & 140 & 5.8 & 7.7 & $2.50 \times 10^{4}$ & 7 \\
\hline GF & HI $(45 \%)$ & 95 & 208 & 2.3 & 10.9 & $0.15 \times 10^{4}$ & 18 \\
\hline GF & AsAc & 80 & 150 & 20.0 & 1.9 & $0.08 \times 10^{4}$ & 24 \\
\hline GF & Thermal reduction & 220 & $115 \pm 19$ & $3.1 \pm 0.7$ & $9.0 \pm 2$ & $0.028 \times 10^{4}$ & 36 \\
\hline GF & $\mathrm{NaBH}_{4}$ & 90 & ca. 8 & $2.8 \pm 1.7$ & - & $2.14 \times 10^{4}$ & 46 \\
\hline
\end{tabular}

graphene filaments can be obtained by applying very high temperature thermal annealing to substitute carbon fiber properties. ${ }^{11,34,37}$ However, the application of high temperature thermal annealing approaching up to $3000{ }^{\circ} \mathrm{C}$ to reduce GOFs requires large investment and also consumption of large amounts of inert gases which are very expensive. Our process conditions avoid such drawbacks for the GFs, which can conduct electricity and can be used in many practical applications.

\section{Conclusions}

The effect of temperature of isopropanol coagulation bath during wet-spinning of graphene oxide filaments on their mechanical properties after formation was investigated and it was found that the decrease of the coagulation bath temperature resulted in better mechanical properties for both GOFs and GFs. The cooler the bath, the slower the inter-diffusion rate occurred between the water molecules present in the GO dispersion and solvent molecules in the bath. Then a better selfassembly of the GO platelets in the filament took place during filament forming. Correspondingly, the cooler the bath, the smaller the filament diameter with a more compact structure was obtained. However, there is an optimum temperature for the coagulation bath since longer filament immersion durations are required for very cold baths. The best tensile strength and Young's modulus values were obtained with GOFs samples which were obtained in isopropanol coagulation bath at $15{ }^{\circ} \mathrm{C}$.

In addition, the effect of the types of the chemical reduction agents to obtain better filament electrical conductivity after reduction of GOFs at medium temperatures was investigated and it was determined that the use of hydroiodic acid/acetic acid mixture resulted in the GFs having the best electrical and also mechanical properties. The inclusion of acetic acid into hydroiodic acid increased the tensile strength $26 \%$ when compared with the plain HI treatment. It was determined that the effect of coagulation bath temperature on the electrical conductivities of final GFs was not important and the best electrical conductivity value was $1.28 \times 10^{4} \mathrm{~S} \mathrm{~m}^{-1}$ which was higher than most of the electrical conductivity results $(0.03-0.86$ $\times 10^{4} \mathrm{~S} \mathrm{~m}^{-1}$ ) for the wet-spun neat GFs reported in the literature. The effect of bath temperature on the mechanical properties of final graphene filaments was very important and a tensile strength value of $234 \pm 26 \mathrm{MPa}$ was obtained when the bath temperature was $15{ }^{\circ} \mathrm{C}$ for an immersion duration of 2 min. This value was higher than most of the previously reported tensile strength values of GFs, which were reduced by using only chemical reducing agents at medium temperatures.

\section{Conflicts of interest}

There are no conflicts to declare.

\section{Acknowledgements}

This work was supported by the Scientific and Technological Research Council of Turkey (TUBITAK) under the project number 115M596.

\section{References}

1 K. S. Novoselov, A. K. Geim, S. V. Morozov, D. Jiang, Y. Zhang, S. V. Dubonos, I. V. Grigorieva and A. A. Firsov, Science, 2004, 306, 183-191.

2 S. Park and R. S. Ruoff, Nat. Nanotechnol., 2009, 4, 217-224. 3 O. C. Compton and S. T. Nguyen, Small, 2010, 6, 711-723.

4 G. Eda, G. Fanchini and M. Chhowalla, Nat. Nanotechnol., 2008, 3, 270-274.

5 D. Li, M. B. Müller, S. Gilje, R. B. Kaner and G. G. Wallace, Nat. Nanotechnol., 2008, 3, 101-105.

6 Y. Xu, K. Sheng, C. Li and G. Shi, ACS Nano, 2010, 4, 43244330.

7 Z. Xu and C. Gao, Nat. Commun., 2011, 2, 571.

8 H. P. Cong, X.-C. Ren, P. Wang and S.-H. Yu, Sci. Rep., 2012, $2,613$.

9 H. Wang, K. Teng, C. Chen, X. Li, Z. Xu, L. Chen, H. Fu, L. Kuang, M. Ma and L. Zhao, Mater. Lett., 2017, 186, 78-81. 10 T. Zhu, K. Teng, J. Shi, L. Chen and Z. Xu, Compos. Sci. Technol., 2016, 123, 276-285.

11 Z. Xu, Y. Liu, X. Zhao, L. Peng, H. Sun, Y. Xu, X. Ren, C. Jin, P. Xu, M. Wang and C. Gao, Adv. Mater., 2016, 28, 6449-6456. 
12 T. Huang, B. Zheng, L. Kou, K. Gopalsamy, Z. Xu, C. Gao, Y. Meng and Z. Wei, RSC Adv., 2013, 3, 23957-23962.

13 S. H. Aboutalebi, R. Jalili, D. Esrafilzadeh, M. Salari, Z. Gholamvand, S. Aminorroaya Yamini, K. Konstantinov, R. L. Shepherd, J. Chen, S. E. Moulton, P. C. Innis, A. I. Minett, J. M. Razal and G. G. Wallace, ACS Nano, 2014, 8, 2456-2466.

14 W. Ma, S. Chen, S. Yang and M. Zhu, RSC Adv., 2016, 6, 50112-50118.

15 M. Huang, L. Wang, S. Chen, L. Kang, Z. Lei, F. Shi, H. Xu and Z. H. Liu, RSC Adv., 2017, 7, 10092-10099.

16 N. He, Q. Pan, Y. Liu and W. Gao, ACS Appl. Mater. Interfaces, 2017, 9, 24568-24576.

17 X. Zheng, K. Zhang, L. Yao, Y. Qiu and S. Wang, J. Mater. Chem. A, 2018, 6, 896-907.

18 S. Chen, W. Ma, Y. Cheng, Z. Weng, B. Sun, L. Wang, W. Chen, F. Li, M. Zhu and H. M. Cheng, Nano Energy, 2015, 15, 642-653.

19 R. Wang, Z. Xu, J. Zhuang, Z. Liu, L. Peng, Z. Li, Y. Liu, W. Gao and C. Gao, Adv. Electron. Mater., 2017, 3, 1600425.

20 Y. Liang, F. Zhao, Z. Cheng, Q. Zhou, H. Shao, L. Jiang and L. Qu, Nano Energy, 2017, 32, 329-335.

21 H. Cheng, C. Hu, Y. Zhao and L. Qu, NPG Asia Mater., 2014, 6, e113.

22 Z. Xu and C. Gao, Acc. Chem. Res., 2014, 47, 1267-1276.

23 Z. Dong, C. Jiang, H. Cheng, Y. Zhao, G. Shi, L. Jiang and L. Qu, Adv. Mater., 2012, 24, 1856-1861.

24 J. Li, J. Li, L. Li, M. Yu, H. Ma and B. Zhang, J. Mater. Chem. A, 2014, 2, 6359-6362.

25 X. Li, T. Zhao, K. Wang, Y. Yang, J. Wei, F. Kang, D. Wu and H. Zhu, Langmuir, 2011, 27, 12164-12171.

26 E. Y. Jang, J. Carretero-González, A. Choi, W. J. Kim, M. E. Kozlov, T. Kim, T. J. Kang, S. J. Baek, D. W. Kim, Y. W. Park, R. H. Baughman and Y. H. Kim, Nanotechnology, 2012, 23, 235601.

27 Z. Xu, Z. Li, H. Sun and C. Gao, Adv. Mater., 2013, 25, 32493253.

28 B. Fang, L. Peng, Z. Xu and C. Gao, ACS Nano, 2015, 9, 52145222.

29 T. Wu, B. Zhou, T. Zhu, J. Shi, Z. Xu, C. Hu and J. Wang, RSC Adv., 2015, 5, 7880-7889.
30 Z. Xu and C. Gao, ACS Nano, 2011, 5, 2908-2915.

31 L. Chen, Y. L. He, S. G. Chai, H. Qiang, F. Chen and Q. Fu, Nanoscale, 2013, 5, 5809-5815.

32 C. Xiang, C. C. Young, X. Wang, Z. Yan, C. C. Hwang, G. Cerioti, J. Lin, J. Kono, M. Pasquali and J. M. Tour, Adv. Mater., 2013, 25, 4592-4597.

33 Z. Xu, H. Sun, X. Zhao and C. Gao, Adv. Mater., 2013, 25, 188193.

34 G. Xin, T. Yao, H. Sun, S. M. Scott, D. Shao, G. Wang and J. Lian, Science, 2015, 349, 1083-1087.

35 Y. S. Kim, J. H. Kang, T. Kim, Y. Jung, K. Lee, J. Y. Oh, J. Park and C. R. Park, Chem. Mater., 2014, 26, 5549-5555.

36 R. Jalili, S. H. Aboutalebi, D. Esrafilzadeh, R. L. Shepherd, J. Chen, S. Aminorroaya-Yamini, K. Konstantinov, A. I. Minett, J. M. Razal and G. G. Wallace, Adv. Funct. Mater., 2013, 23, 5345-5354.

37 X. Zheng, L. Yao, X. Mei, S. Yu, W. Zhang and Y. Qiu, J. Mater. Sci., 2016, 51, 9889-9901.

38 D. C. Marcano, D. V. Kosynkin, J. M. Berlin, A. Sinitskii, Z. Sun, A. Slesarev, L. B. Alemany, W. Lu and J. M. Tour, ACS Nano, 2010, 4, 4806-4814.

39 R. Cruz-Silva, A. Morelos-Gomez, H. I. Kim, H. K. Jang, F. Tristan, S. Vega-Diaz, L. P. Rajukumar, A. L. Elías, N. Perea-Lopez, J. Suhr, M. Endo and M. Terrones, ACS Nano, 2014, 8, 5959-5967.

40 C. Xu, X. Shi, A. Ji, L. Shi, C. Zhou and Y. Cui, PLoS One, 2015, 10, e0144842.

41 C. Y. Su, Y. Xu, W. Zhang, J. Zhao, X. Tang, C. H. Tsai and L. J. Li, Chem. Mater., 2009, 21, 5674-5680.

42 S. Pei, J. Zhao, J. Du, W. Ren and H. M. Cheng, Carbon, 2010, 48, 4466-4474.

43 I. K. Moon, J. Lee, R. S. Ruoff and H. Lee, Nat. Commun., 2010, 1, 73.

44 J. Hao, F. An, Y. Yu, P. Zhou, Y. Liu and C. Lu, J. Appl. Polym. Sci., 2017, 134, 44390.

45 A. F. Ismail, M. A. Rahman, A. Mustafa and T. Matsuura, Mater. Sci. Eng., A, 2008, 485, 251-257.

46 R. Bakhtiari, S. Ghobadi, E. N. Gulluoglu, L. I. Sanll, S. A. Gursel and E. Ozden-Yenigun, RSC Adv., 2017, 7, 26735-26744.

47 X. Hu, Z. Xu, Z. Liu and C. Gao, Sci. Rep., 2013, 3, 2374. 